\title{
Tetracycline Actions Relevant to Rosacea Treatment
}

\author{
H.C. Korting C.Schöllmann \\ Department of Dermatology and Allergology, Ludwig-Maximilians-Universität, Munich, Germany
}

\section{Key Words}

Rosacea $\cdot$ Tetracyclines $\cdot$ Doxycycline $\cdot$ Inflammation $\cdot$ Matrix metalloproteinases $\cdot$ Cytokines $\cdot$ Reactive oxygen species $\cdot$ Angiogenesis

\begin{abstract}
Until today, the pathogenesis of rosacea is not known in detail. Yet in recent years evidence has been accumulating that rosacea with its common symptoms such as inflammatory lesions, erythema, telangiectasia, phymatous changes, and ocular symptoms is of inflammatory nature. Tetracycline derivatives like doxycycline successfully used in the treatment of skin diseases like acne and rosacea seem to inhibit different inflammatory pathways involved in the pathogenesis by various modes of action. Although data for skin diseases are relatively scanty, the following modes of action of tetracyclines seem to be most relevant for an effective treatment of acne and rosacea: inhibition of matrix metalloproteinases, downmodulation of cytokines, inhibition of cell movement and proliferation, inhibition of granuloma formation, inhibition of reactive oxygen species, nitric oxide, and angiogenesis, whereas inhibition of phospholipase A2 seems to be of lower importance. The role of the saprophytic mite Demodex folliculorum remains to be clarified. Additional studies are necessary to further elucidate how tetracyclines work in rosacea treatment.

Copyright $\odot 2009$ S. Karger AG, Basel
\end{abstract}

\section{Pathophysiology of Rosacea}

Rosacea is a common chronic, often underdiagnosed, skin disease of uncertain etiology. The various clinical manifestations associated with this disease most frequently occur in the light-skinned Caucasian population, and in persons between 30 and 50 years of age $[1,2]$. Until now the conventional wisdom has been that rosacea is more common in women than in men [2], whereas a recent analysis based on a cross-sectional study of rosacea (1995-2002) in 50,235 outpatients indicates that overall both sexes are equally affected by the disease [3]. The dermatological condition of rosacea mainly affects the cheek, nose, chin and central forehead. Furthermore, the disease may be linked to ocular manifestations [4]. The earliest symptoms associated with rosacea are intermittent, central facial flushing and erythema. More than $90 \%$ of the patients with rosacea experience flushing with episodes lasting up to 30 min occurring daily in more than $60 \%$ of the affected individuals [5-7]. With repetitive cycles, flushing becomes more prominent and facial redness deepens, in the end repeated episodes of facial flushing may cause persistent erythema and telangiectasia [8]. Many patients suffer from a stinging pain associated with episodes of flushing which can appear unpredictably or in relation to environmental, chemical, food or emotional triggers, especially exposure to sun [1].

\section{KARGER}

Fax +4161306 1234 E-Mail karger@karger.ch www.karger.com
Prof. Dr. med. Hans Christian Korting

Department of Dermatology and Allergology, Ludwig Maximilian University

Frauenlobstrasse 9-11, DE-80337 Munich (Germany)

Tel. +49 895160 6154, Fax +49 8951606153

E-Mail H.C.Korting@lrz.uni-muenchen.de 
Until today, the pathogenesis of rosacea is not known in detail. Yet in recent years evidence has been accumulating that rosacea is of inflammatory nature even though most data have their origin in diseases related to rosacea, namely acne vulgaris and, most commonly, periodontitis. For example, in biopsies of inflammatory lesions from patients with acne an increase in proinflammatory cytokines like tumor necrosis factor- $\alpha$ (TNF- $\alpha$ ) and interleukin-1 $\beta$ (IL-1 $\beta$ ) could be demonstrated [9]. These cytokines trigger a chain of reactions, such as the release of matrix metalloproteinases (MMPs), especially MMP-1, -3 and -9 , involved in the degradation of extracellular matrix and inflammatory damage in turn supporting the development of papulopustular lesions $[10,11]$. The fact that such lesions occur in rosacea as well supports the thesis of an inflammatory pathophysiology of this disease. Anyway, 2 further mediators of inflammation, reactive oxygen species (ROS) and nitric oxide (NO), have been shown to play a role in rosacea pathophysiology $[12,13]$. It has in particular been shown that patients with severe rosacea have an impaired defense capacity against the proinflammatory and damaging effects of ROS [11, 14]. Reactive nitrogen intermediates like NO in turn are responsible for vasodilatation possibly leading to increased vessel permeability and edema and supporting erythema and telangiectasia [11]. Flushing is caused by increased cutaneous blood flow because of capillary dilatations and is believed to be under vasomotor control [8]. It seems to arise from a dysregulation in the cutaneous vasomotor response, which, triggered by neurogenic, hormonal, thermal, topical or other stimuli, leads to an abnormal and persistent dilatation of blood vessels [15]. It is worth discussing that flushing - at least in the erythematotelangiectatic subtype of rosacea - may be due in part to an abnormal expression, function, distribution or responsiveness of $\alpha$-adrenergic receptors [15].

A possible link between Helicobacter pylori infections and rosacea is discussed in the literature as well [11]. Several authors have also proposed that microscopic saprophytic mites - known as Demodex folliculorum - play a pathogenic role in papulopustular rosacea [16-21]. Mites increased in number may be involved in the pathogenesis of rosacea by provoking inflammatory or allergic reactions, by mechanical blockage of follicles or by acting as vectors for micro-organisms $[17,22]$. It has been suggested that a density of $D$. folliculorum $>5 / \mathrm{cm}^{2}$ as determined with the standardized skin surface biopsy could be regarded as pathogenic [18], but other observations suggest that increased density of $D$. folliculorum, at least in peri- oral dermatitis, is a secondary phenomenon, associated with previous treatment with topical steroids [23].

Most recently it could be shown that symptoms of rosacea are exacerbated by factors that trigger innate immune responses, such as the release of cathelicidin antimicrobial peptides. These peptides are produced and secreted by keratinocytes [24]. Clearly, there is a role of cathelicidin in skin inflammatory responses [25], especially for cathelicidin LL-37 [24]. Individuals with rosacea express cathelicidin at abnormally high levels in their facial skin, and specific proteolytically processed forms of cathelicidin peptides found in rosacea differ from those in normal individuals [25]. This means that in the pathogenesis of rosacea, cathelicidin peptides are abnormally processed to forms that induce inflammation [26].

If changes in the skin $\mathrm{pH}$ play a role in pathophysiology, as it is reported for several skin diseases like irritant contact dermatitis, atopic dermatitis, ichthyosis or acne vulgaris [27], remains to be clarified.

In summary, there is good evidence that all the stigmata of rosacea are manifestations of an inflammatory process. It is highly probable that inflammatory pathways involved in the pathogenesis of this disease concomitantly contribute to the common complaints such as inflammatory lesions sensu stricto, erythema, telangiectasia, phymatous changes and ocular symptoms [28]. This view is supported by the fact that drugs like doxycycline successfully used in the treatment of rosacea inhibit various pathways of inflammation finally leading to an improvement of symptoms [9, 13, 29-31]. Furthermore, the anti-inflammatory properties of doxycycline strongly correspond to the pathophysiologic inflammatory mechanisms related to rosacea [31].

\section{Tetracyclines and Their Role in the Treatment of Rosacea}

Tetracyclines are broad-spectrum antibiotics that have been used in the treatment of rosacea for decades [9]. Treatment of papulopustular rosacea with tetracycline requires a 3- or 4-week regimen to achieve substantial improvement [32]. Tetracycline (250-1,000 mg per day), doxycycline (100-200 mg per day and - as demonstrated recently - also 20-40 mg per day) and minocycline (50$100 \mathrm{mg}$ per day) are the most commonly applied compounds [33]. Until recently, the use of oral tetracyclines for rosacea was mainly based on clinical experience and a limited number of placebo-controlled studies $[9,34,35]$. 
However, most recent studies were carried out on the basis of a sufficiently large number of patients and can therefore be considered to be of high quality [31].

Today, the effectiveness of tetracyclines in skin diseases is considered to depend on their nonantibiotic actions, though originally developed as antibacterial drugs. Tetracyclines, besides acting as antibiotics, may also affect inflammation [31,36], cell proliferation [37], apoptosis $[38,39]$ and angiogenesis $[40]$ by variable mechanisms of action. In particular, it is the ability of tetracyclines to reduce the inflammatory response that significantly contributes to the clinical effectiveness for various indications like rosacea [30]. However, the observation that tetracyclines under ex vivo conditions augment the expression of cyclo-oxygenase- 2 and increase prostaglandin E2 production [41] is not in line with the data described before because cyclo-oxygenase- 2 and prostaglandin E2 activation are known to propagate inflammatory processes [42].

Today, second-generation tetracyclines, including minocycline and especially doxycycline, are most frequently and most successfully used in the treatment of rosacea. In comparison with their parent drug, the new generation of tetracyclines has an improved bioavailability, a longer elimination half-life and can be taken with food which minimizes gastrointestinal side effects [43]. A big advantage over the parent drug is that these substances are helpful for rosacea patients already at a subantimicrobial yet anti-inflammatory dose [31]. Thus, an effective and tolerable long-term therapy is feasible without running the risk of long-term antibiosis, i.e. undesired adverse effects such as gastrointestinal distress, candidal vulvovaginitis and/or the development of antibiotic-resistant micro-organisms [44].

Subantimicrobial-dose doxycycline is the only tetracycline approved in the USA for long-term use for up to 12 months [33]. The dosage of $20 \mathrm{mg}$ of doxycycline hyclate twice daily or $40 \mathrm{mg}$ once daily has been shown to be effective in the treatment of papulopustular rosacea with an excellent risk-benefit ratio. It has in particular been found that a $40-\mathrm{mg}$ controlled-release formulation of doxycyline, applied once daily as Oracea ${ }^{\mathrm{TM}}$, conferred peak anti-inflammatory efficacy in the treatment of rosacea [45]. At subantimicrobial doses, long-term use of anti-inflammatory-dose doxycyline might not propagate bacterial resistance $[33,44,46]$. Two phase III, parallelgroup, multicenter, randomized, double-blind, placebocontrolled studies have in fact further demonstrated the efficacy and safety of a 16-week treatment with anti-inflammatory doxycycline (40 $\mathrm{mg}$ once daily) in patients with rosacea [31]. This medication led to a significant reduction in inflammatory lesions [31]. Hence anti-inflammatory doxycycline $(40 \mathrm{mg})$ applied once daily must be regarded to be a promising therapy strategy for papulopustular rosacea - besides topical therapies with metronidazole or azelaic acid. However, to our knowledge it is not known if tetracyclines, in particular doxycycline, are able to improve the symptom flushing, whereas topical application of selective $\alpha 1$-adrenergic receptor agonists like oxymetazoline seems to be successful in this regard [15]. This finding is in accordance with the belief that flushing may be due, at least partly, to an abnormal expression, function, distribution or responsiveness of $\alpha$ adrenergic receptors [15].

\section{Tetracycline Derivatives, Especially Doxycycline, and Their Mode of Action}

\section{Inhibition of MMPs}

In the 1980s, Golub et al. [47] discovered a novel nonantimicrobial property of tetracyclines. They found that tetracyclines have the ability to inhibit the activity of interstitial MMPs from a variety of cells like neutrophils and macrophages and tissues such as skin [47, 48]. MMPs are zinc-dependent enzymes significantly involved in the remodeling of connective tissue. They are secreted by resident and inflammatory cells that collectively degrade most of the constituent macromolecules of the extracellular matrix. These enzymes with their subgroups of collagenases (MMP-1, MMP-8, MMP-13) and gelatinases (MMP-2, MMP-9) play an important role in many inflammatory diseases, wound healing, embryogenesis, tumor invasion and angiogenesis [30, 49]. Furthermore, MMPs contribute to cytokine (i.e. IL-1 $\beta$ )-induced vascular dysfunction as could be shown in vitro and in an in vivo model of acute inflammation [50] (fig. 1). Later it has been observed that tetracycline inhibition of MMPs differs between species, tissues and even cells from the same tissue [51]. Furthermore, it could be shown that the members of the tetracycline family differ in their potency to inhibit extracellular collagenase activity, with the semisynthetic drugs doxycycline and minocycline being more potent than tetracycline itself $[48,52]$.

In vitro and in vivo studies report that tetracycline derivatives are able to inhibit the activity of various MMPs playing a role in the breakdown of the extracellular matrix, including MMP-13 (collagenase-3), MMP-8 (collagenase-2), MMP-1 (collagenase-1), MMP-2 (gelatinase A), MMP-9 (gelatinase B) and MMP-12 (macro- 


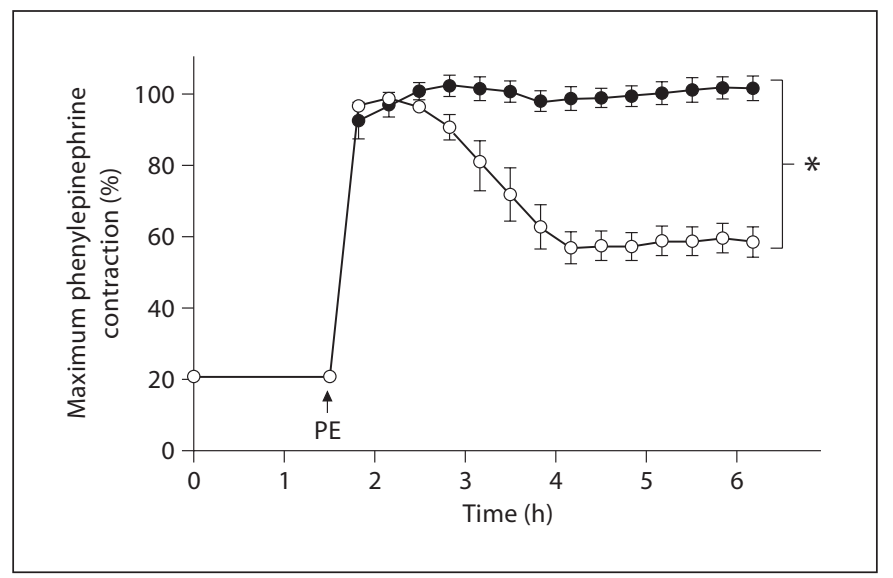

Fig. 1. Application of MMP inhibitor doxycycline (30 $\mu \mathrm{M})$ abolishes the spontaneous loss of phenylepinephrine-induced vascular tone of rat aortae in vitro. The response of aortic rings taken from normal rats to phenylepinephrine is shown. Phenylepinephrine was added after $1.5 \mathrm{~h}$ of equilibration and then rings were treated with doxycycline ( $30 \mu \mathrm{M}$; black circles) or double-distilled $\mathrm{H}_{2} \mathrm{O}$ (white circles) vehicle (from Lalu et al. [50], with permission).

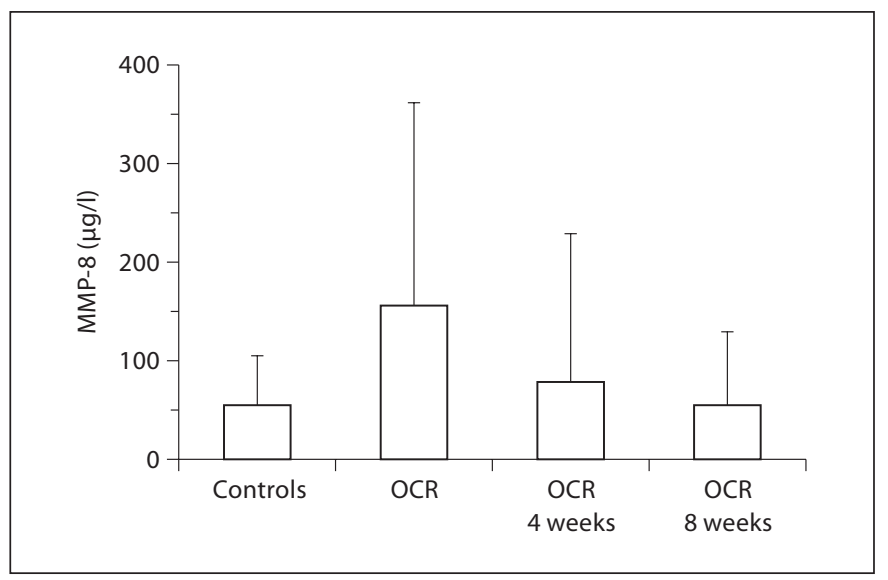

Fig. 2. Tear fluid MMP-8 concentration in controls, as well as ocular rosacea (OCR) patients before treatment and after 4 and 8 weeks of oral doxycycline regimen. Means \pm SD are presented (from Määtä et al. [63], with permission).

phage metalloelastase) $[11,31,53,54]$. For some MMPs it has been shown that, in addition to inhibiting MMP activity, tetracycline derivatives like doxycycline may also reduce enzyme expression at the transcriptional level [55].

While the inhibitory effects of low-dose doxycycline on MMPs are described in detail in the context of the man- agement of periodontitis [56-60], available data for skin diseases like acne or rosacea are relatively scanty. However, there are data indicating that MMP-9 can be inhibited by tetracycline derivatives as well probably due to reduction in the chemotaxis of neutrophils, which are known to store MMP-9 [30]. The inhibitory effect of doxycycline on MMP-9 levels could be approved by studies using an experimental dry-eye model in mice [61]. The studies of Kim et al. [62] showing that doxycycline inhibits the TGF$\beta 1$-induced production of MMP- 9 in cultured corneal epithelial cells as well suggest hat this finding might be relevant to the pathogenesis in ocular rosacea $[49,62]$. More evidently, recent work even suggests that high levels of MMP-8 in the tear fluid are correlated with ocular rosacea and that low-dose doxycycline is able to lower the pathologically increased levels in these patients. Määtä et al. [63] actually found that the mean MMP-8 concentrations were significantly higher in patients with ocular rosacea (157 $\mu \mathrm{g} / \mathrm{ml})$ than in normal subjects $(53 \mu \mathrm{g} / \mathrm{ml})$ but decreased to 79 and $53 \mu \mathrm{g} / \mathrm{ml}$ in patients with ocular rocacea after 4 and 8 weeks of treatment with low-dose doxycycline, respectively (fig. 2). The reduction in MMP-8 levels was related to a relief in the patients' subjective symptoms [63]. Up to now, doxycycline hyclate is the only drug whose properties to inhibit MMPs have sufficed for approval of clinical use [64], but the exact mode of action on the molecular level is still unknown. Recent work indicates that doxycycline does not directly inhibit MMPs or the synthesis/secretion of these proteases. Though orally administered doxycycline caused a disappearance of the MMPs indicative of disease progression in patients with superficial ocular diseases, especially rosacea, no doxycyline could be detected in their tear samples [65]. Thus, doxycycline seems to influence MMPs in an indirect way.

\section{Inhibition of Phospholipase A2}

Extracellular phospholipase A2 (PLA2) plays an important role in articular and extra-articular inflammatory processes [66]. The tetracyclines minocycline and, to a lesser extent, doxycycline have been shown to be inhibitory to both pancreatic and nonpancreatic PLA2 in vitro [66]. However, the doxycycline concentration needed for in vitro inhibition of the enzyme activity in serum from patients with acute necrotizing pancreatitis is higher than that achieved after intravenous injections in clinical use [67]. Thus, up to now the importance of PLA2 inhibition by doxycycline in vivo and especially in skin diseases like rosacea remains unclear, particularly with regard to the finding that in tears of patients with ocular rosacea and, more specifically, in patients with dry eyes 
the content of PLA2 of group IIA is significantly lower compared to normal controls [68].

\section{Downmodulation of Cytokines}

Inflammatory lesions from acne patients contain increased levels of proinflammtory cytokines like TNF- $\alpha$ and IL- $1 \beta$ postulated to be involved in the inflammatory cascade of acne [9] and triggering chemical responses like the release of certain MMPs [13]. There is evidence that tetracyclines at subantimicrobial doses reduce inflammation by their downregulating properties [36], especially with respect to TNF- $\alpha$ and IL-1 $\beta$ [69], IL-8 [70] and IL-10 [71]. Furthermore, low-dose doxycycline seems to be able to normalize lowered levels of cytokines necessary for repair and regeneration. In patients with severe generalized chronic periodontitis, subantimicrobialdose doxycycline increases the levels of TGF- $\beta 1$ in the gingival crevicular fluid and by this means contributes to the inhibition of connective tissue breakdown [72]. For rosacea no corresponding studies are available so far, but it is to be assumed that the described mechanisms are, at least partly, transferable to this disease as well.

\section{Inhibition of Cell Movement and Proliferation}

The migration of white blood cells is an early and important event in the process of inflammation. Data from the late 1970s and 1980s show that tetracyclines are able to interfere with neutrophil function [37], especially with neutrophil movement, at least in vitro [73]. Tetracyclines have also been found to be able to inhibit the mitogen-induced proliferative responses of human lymphocytes in vitro by blockage of blast formation, with doxycycline being the most potent inhibitor among 3 tested tetracycline analogues [37]. Gabler et al. [74] could show that the chelation of intracellular calcium, which, as a second messenger, is important for the regulation of cell movement, is involved in the mechanism of inhibition. Besides migration, tetracyclines are also able to inhibit neutrophil-mediated superoxide anion synthesis and degranulation, i.e. mechanisms which may also be involved in the inflammatory process [75]. Lately, Gabler and Tsukuda [76] found that doxycycline also exerts a slight inhibitory effect on neutrophil adherence to surfaces coated with differing proteins, which interestingly could only be observed at low drug concentrations of doxycycline, while tetracycline inhibited leukocyte adherence in a dose-related manner.

\section{Inhibition of Granuloma Formation}

Granulomatous inflammation is a common component of many diseases, including rosacea. Tetracyclines have been shown to inhibit granuloma formation of human peripheral blood mononuclear cells incubated with dextrin beads [77]. In this in vitro system doxycycline was proven to have a 10 -fold stronger inhibitory activity than the parent drug tetracycline. Protein kinase $\mathrm{C}$ may be involved in the described mechanism of inhibition because tetracyclines caused a dose-dependent inhibition of protein kinase $\mathrm{C}$ in the same order of relative potency as was found for the inhibition of granuloma formation [77].

\section{Inhibition of ROS}

Evidence has been accumulating that ROS released by inflammatory cells such as neutrophils [12] are involved in the pathogenesis of rosacea. Patients with rosacea have higher ROS levels than healthy controls [78]. Furthermore, markers of the antioxidant defense system are inversely correlated to the clinical manifestations of rosacea. This means that in patients with mild rosacea (stage I or II) the superoxide dismutase activity was higher than in healthy controls while the malondialdehyde level (a biomarker for oxidative stress [79]) did not differ from controls, whereas in the severe involvement group (rosacea stage III) the superoxide dismutase activity was lower than in the control group coupled to an increased level of malondialdehyde [14]. These findings suggest that in mild rosacea the antioxidant defense system is activated in order to keep the ROS levels constant. In severer disease, the capacity of the antioxidant system is exhausted, leading to high levels of ROS. This belief is supported by recent data showing a higher ferritin expression in the skin, higher serum peroxide levels and significantly lowered total antioxidative potential serum levels in patients with rosacea compared with healthy control subjects, indicating the onset of systemic oxidative stress in patients with rosacea [80]. These findings support the 'antioxidant system defect hypothesis' in rosacea patients, i.e. a reduced capacity to counter the negative effects of ROS, thus experiencing an increased inflammatory response $[13,14]$. This may also explain the pathogenetic association between rosacea and photodamage, since long-term sun exposure is known to cause an increased generation of ROS, which, in turn, subsequently activate MMPs and in this way trigger the inflammation pathway $[13,31]$.

In vitro studies suggest that tetracyclines, especially doxycycline, show antioxidant effects by reducing ROS $[30,60]$. In vitro data propose that the antioxidant effect of these drugs does not originate from their capability to directly scavenge ROS but from their influence on white blood cells [81]. However, the exact mechanism of action 
of tetracyclines towards white blood cells, especially neutrophils, is still unknown $[12,82]$. The tetracycline-induced reduction in ROS activity seems to be linked to a decreased degradation of extracellular matrix associated with a reduced activation of pro-MMPs [31]. Thus, the antioxidant activity of tetracyclines may also benefit other mechanisms of inflammation.

\section{Inhibition of $\mathrm{NO}$}

The overproduction of NO has been implicated in the pathogenesis of various inflammatory diseases [83]. NO secreted by endothelial cells and keratinocytes in response to UVA and UVB radiation [84] is thought to incite the symptoms of rosacea as well [13]. NO seems to be involved in vascular changes, i.e. vasodilation, which, in the case of rosacea, may contribute to increased vessel permeability, erythema, edema and telangiectatic symptoms [13]. The increase in NO in response to UVA and UVB irradiation [84] again refers to the widely accepted relationship between sun exposure and the pathogenesis of rosacea.

Tetracyclines have been shown to inhibit the expression of nitric synthases under ex vivo conditions, with doxycycline being at least as effective as minocycline [83]. Furthermore, doxycycline has been shown to be involved in the posttranscriptional regulation of inducible $\mathrm{NO}$ synthetase mRNA in murine macrophages [85]. All these preliminary results suggest that tetracyclines, especially doxycycline, may have beneficial effects in the treatment of inflammatory diseases where excess NO has been implicated in the pathophysiology, as also seems to be the case with rosacea.

\section{Inhibition of Angiogenesis}

Angiogenesis seems to be involved in the pathogenesis of rosacea $[19,86]$ and to be linked to MMP activity [36]. In patients with rosacea, significantly increased dermal expression of the potent angiogenesis factor vascular endothelial growth factor in lesional versus nonlesional skin ( 88.9 vs. $55.6 \%$, respectively) was observed. Furthermore, more microvessels were observed in lesional compared to clinically nonlesional skin, and a high microvessel density was found to be correlated with granuloma formation in the skin [19]. There was also a correlation between high vascular density and the presence of ocular manifestations [19].

Up to now, no study is available showing a direct or indirect antiangiogenic effect of tetracyclines in the pathogenesis of rosacea. Yet in a mouse model, doxycycline has been found active in suppressing MMP-associ- ated central nervous system angiogenesis $[49,87]$. In this model, doxycycline turned out to be a potent inhibitor of angiogenesis induced by vascular endothelial growth factor by decreasing the cerebral MMP-9 activity. The decrease in MMP-9 was associated with a reduced microvessel count [87]. More recently, it has been shown that doxycycline attains consistent antiangiogenic effects by inhibiting the migration of smooth-muscle cells playing an important role during angiogenesis and vascular remodeling [88]. There is good evidence that inhibition of smooth-muscle cell migration is caused by the doxycycline-induced upregulation of the MMP inhibitor tissue inhibitor of metalloproteinase-1 [88].

\section{Influence on D. folliculorum}

The saprophytic mite D. folliculorum may play a pathogenic role in papulopustular rosacea [17-21]. Increased density of $D$. folliculorum may be involved in the pathogenesis of rosacea by supporting inflammation, mechanical blockage of follicles or by acting as vectors for microorganisms [17,22], while other authors favour the concept that increased mite density is the consequence of a previous treatment with topical steroids [23]. Up to now, to our knowledge no study showing a relevant direct action of tetracyclines on the density of $D$. folliculorum is available.

\section{Conclusion}

It is highly probable that rosacea is of inflammatory nature. Inflammatory processes involved in the pathogenesis of the disease concomitantly contribute to the common symptoms such as inflammatory lesions in the stricter sense, erythema, telangiectasia, phymatous changes and ocular symptoms. Evidence has been accumulating that tetracycline derivatives like doxycycline successfully used in the treatment of rosacea inhibit inflammatory pathways in skin diseases like acne and rosacea by various modes of action. Although data for acne or rosacea are relatively scanty, the following modes of action of tetracyclines seem to be most relevant to an effective treatment of these skin diseases: inhibition of MMPs, downmodulation of cytokines, inhibition of cell movement and proliferation, granuloma formation, ROS, NO and angiogenesis, whereas inhibition of PLA2 does not seem to matter greatly. The role of the saprophytic mite D. folliculorum remains to be clarified. Nevertheless, future studies will have to show how tetracyclines work in rosacea treatment in detail. 


\section{References}

1 Blount BW, Pelletier AP: Rosacea: a common yet commonly overlooked condition. Am Fam Physician 2002;66:435-440, 442.

2 Berg M, Liden S: An epidemiological study of rosacea. Acta Derm Venereol 1989;69:419423.

3 Kyriakis KP, Palamaras I, Terzoudi S, Emmanuelides S, Michailides C, Pagana G: Epidemiologic aspects of rosacea. J Am Acad Dermatol 2005;53:918-919.

4 Eiseman AS: The ocular manifestations of atopic dermatitis and rosacea. Curr Allergy Asthma Rep 2006;6:292-298.

5 Dahl MV: Pathogenesis of rosacea. Adv Dermatol 2001; 17:29-45.

6 Rebora A: The management of rosacea. Am J Clin Dermatol 2002;3:489-496.

7 Brinnel H, Friedel J, Caputa M, Cabanac M, Grosshans E: Rosacea: disturbed defense against brain overheating. Arch Dermatol Res 1989;281:66-72.

8 Yale SH, Vasudeva S, Mazza JJ, Rolak L, Arrowood J, Stichert S, Stratman ES: Disorders of flushing. Compr Ther 2005;31:59-71.

9 Pelle MT, Crawford GH, James WD: Rosacea. II. Therapy. J Am Acad Dermatol 2004; 51:499-512.

10 Kang S, Cho S, Chung JH, Hammerberg C, Fisher GJ, Voorhees JJ: Inflammation and extracellular matrix degradation mediated by activated transcription factors nuclear factor- $\kappa \mathrm{B}$ and activator protein-1 in inflammatory acne lesions in vivo. Am J Pathol 2005;166:1691-1699.

11 Golub LM, Lee HM, Ryan ME, Giannobile WV, Payne J, Sorsa T: Tetracyclines inhibit connective tissue breakdown by multiple non-antimicrobial mechanisms. Adv Dent Res 1998;12:12-26.

12 Jones D: Reactive oxygen species and rosacea. Cutis 2004;74(suppl 3):17-20, 32-34.

13 Bikowski J: Examining inflammation as a common factor in theories of rosacea pathophysiology. www.rosacea.org (last accessed November 24, 2008)

14 Oztas MO, Balk M, Ogüs E, Bozkurt M, Ogüs IH, Ozer N: The role of free oxygen radicals in the aetiopathogenesis of rosacea. Clin Exp Dermatol 2003;28:188-192.

15 Shanler SD, Ondo AL: Successful treatment of the erythema and flushing of rosacea using a topically applied selective $\alpha 1$-adrenergic receptor agonist, oxymetazoline. Arch Dermatol 2007;143:1369-1371.

16 Szlachic A: The link between Helicobacter pylori infection and rosacea. J Am Acad Dermatol Venereol 2002;16:328-333.

17 Bonnar A, Eusta P: The Demodex mite population in rosacea. J Am Acad Dermatol 1993; 28:443-448.

18 Forton F, Seys B: Density of Demodex folliculorum in rosacea: a case-control study using standardized skin-surface biopsy. Br J Dermatol 1993;128:650-659.
19 Aroni K, Tsagroni E, Kavantzas N, Patsouris E, Ioannidis E: A study of the pathogenesis of rosacea: how angiogenesis and mast cells may participate in a complex multifactorial process. Arch Dermatol Res 2008;300:125131.

20 Hsu CK, Hsu MML, Lee JYY: Demodicosis a clinicopathological study. J Am Acad Dermatol 2009;60:453-462.

21 Dhingra KK, Saroha V, Gupta P, Khurona N: Demodex-associated dermatologic conditions - a coincidence or an etiological correlate: review with a report of a rare case of sebaceous adenoma. Pathology Res Pract 2009;205:423-426.

22 Lacey N, Delaney S, Kavanagh K, Powell FC: Mite-related antigens stimulate inflammatory cells in rosacea. Br J Dermatol 2007;157: 474-478.

23 Dolenc-Voljč M, Pohar M, Lunder T: Density of Demodex folliculorum in perioral dermatitis. Acta Derm Venereol 2005;85:211-215.

24 Schauber J, Ruzicka T, Rupec RA: Cathelicidin LL-37: ein zentraler Faktor in der Pathogenese entzündlicher Dermatosen? Hautarzt 2008;59:72-74.

25 Yamasaki K, Di Nardo A, Bardan A, Murakami $\mathrm{M}$, Ohtake $\mathrm{T}$, Coda $\mathrm{A}$, Dorschner RA, Bonnart C, Descarques P, Hoynanian A, Morhenn VB, Gallo RL: Increased serine protease activity and cathelicidin promotes skin inflammation in rosacea. Nat Med 2007;13:975-980.

26 Schauber J, Gallo RL: Antimicrobial peptides and the skin immune defense system. Allergy Clin Immunol 2008;122:261-266.

27 Schmid-Wendtner MH, Korting HC: The $\mathrm{pH}$ of the skin surface and its impact on the barrier function. Skin Pharmacol Physiol 2006; 19:296-302.

28 Korting HC, Schöllmann C: Current topical and systemic approaches to treatment of rosacea. J Eur Acad Dermatol Venereol 2009; 23:876-882.

29 Thiboutot D, Thieroff-Ekerdt R, Graupe K: Efficacy and safety of azelaic acid (15\%) gel as a new treatment for papulopustular rosacea: results from two vehicle-controlled, randomized phase III studies. J Am Acad Dermatol 2003;48:836-845.

30 Sapadin AN, Fleischmajer R: Tetracyclines: nonantibiotic properties and their clinical implications. J Am Acad Dermatol 2006;54: 258-265

31 Del Rosso JQ, Webster GF, Kackson M, Rendon M, Rich P, Torok H, Bradshaw M: Two randomized phase III clinical trials evaluating anti-inflammatory dose doxycycline (40-mg doxycycline, USP capsules) administered once daily for treatment of rosacea. J Am Acad Dermatol 2007;56:791-802.

32 Sneddon IB: A clinical trial of tetracycline in rosacea. Br J Dermatol 1966;78:649-652.

33 Baldwin HE: Systemic therapy of rosacea. Skin Therapy Lett 2007; 12:1-5.

34 Del Rosso JQ: Medical treatment of rosacea with emphasis on topical therapies. Expert Opin Pharmacother 2004;5:5-13.
35 Bikowski JB, Goldman MP: Rosacea: where are we now? J Drugs Dermatol 2004;3:251261.

36 Bikowski JB: Subantimicrobial dose doxycycline for acne and rosacea. Skinmed 2003;2: 234-245

37 Thong YH, Ferrante A: Inhibition of mitogen-induced human lymphocyte proliferative responses by tetracycline analogues. Clin Exp Immunol 1979;35:443-446.

38 Sandler C, Nurmi K, Lindstedt KA, Sorsa T, Golub LM, Kovanen PT, Eklund KK: Chemically modified tetracyclines induce apoptosis in cultured mast cells. Int Immunopharmacol 2005;5:1611-1621.

39 Onoda T, Ono T, Dhar DK, Yamanoi A, Nasague $\mathrm{N}$ : Tetracyline analogues (doxycycline and COL-3) induce caspase-dependent and -independent apoptosis in human colon cancer cells. Int J Cancer 2006;118:1309-1315.

40 Fainaru O, Adini I, Benny O, Bazinet L, Pravda E, D’Amato R, Folkman J: Doxycycline induces membrane expression of VEcadherin on endothelial cells and prevents vascular permeability. FASEB J 2008;22: 3728-3735.

41 Attur MG, Patel RN, Patel ND, Abramson SB, Amin AR: Tetracycline up-regulates COX-2 expression and prostaglandin E2 production independent of its effect on nitric oxide. J Immunol 1999;162:3160-3167.

42 Samuelsson B, Morgenstern R, Jakobsson PJ: Membrane prostaglandin E synthase-1: a novel therapeutic target. Pharmacol Rev 2007;59:207-224

43 Maibach H: Second-generation tetracyclines, a dermatological overview: clinical uses and pharmacology. Cutis 1991;48:411417.

44 Berman B, Perez OA, Zell D: Update on rosacea and anti-inflammatory-dose doxycycline. Drugs Today (Barcelona) 2007;43:2734.

45 Theobald K, Bradshaw M, Leyden J: Anti-inflammatory dose doxycycline (40 mg controlled-release) confers maximum anti-inflammatory efficacy in rosacea. Skinmed 2007;6:221-226.

46 Walker C, Puumala S, Golub LM, Stoner JA, Reinhardt RA, Lee HM, Payne JB: Subantimicrobial dose doxycycline effects on osteopenic bone loss: microbiologic results. J Periodontol 2007;78:1590-1601.

47 Golub LM, Ramamurthy N, McNamara TF, Gomes B, Wolff M, Casina A, Kapoor A, Zambon J, Ciancio S: Tetracyclines inhibit tissue collagenase activity: a new mechanism in the treatment of periodontal disease. J Periodont Res 1984;19:651-655.

48 Golub LM, Ramamurthy NS, McNamara TF, Greenwald RA, Rifkin BR: Tetracyclines inhibit connective tissue breakdown: new therapeutic implications for an old family of drugs. Crit Rev Oral Biol Med 1991;2:297322 . 
49 Webster G, Del Rosso JQ: Anti-inflammatory activity of tetracyclines. Dermatol Clin 2007;25:133-135.

50 Lalu MM, Cena J, Chowdhury R, Lam A, Schulz R: Matrix metalloproteinases contribute to endotoxin- and interleukin- $1 \beta$-induced vascular dysfunction. Br J Pharmacol 2006;149:31-42.

51 Vernillo AT, Ramamurthy NS, Golub LM, Rifkin BR: The nonantimicrobial properties of tetracycline for the treatment of periodontal disease. Curr Opin Periodontal 1994;2: 111-118.

52 Golub LM, Soumalainen K, Sorsa T: Host modulation with tetracyclines and their chemically modified analogues. Curr Opin Dent 1992;2:80-90.

53 Bonamigo RR, Bakos L, Edelweiss M, Cartell A: Could matrix metalloproteinase- 9 be a link between Demodex folliculorum and rosacea? J Eur Acad Dermatol Venereol 2005; 19:646-647.

54 Afonso AA, Sobrin L, Monroy DC, Selzer M, Lokeshwar B, Pflugfelder SC: Tear fluid gelatinase $B$ activity correlates with IL-1 $\alpha$ concentration and fluorescein clearance in ocular rosacea. Invest Ophthalmol Vis Sci 1999; 40:2506-2512.

55 Uitto VJ, Firth JD, Nip L, Golub LM: Doxycyline and chemically modified tetracyclines inhibit gelatinase A (MMP-2) gene expression in human skin keratinocytes. Ann NY Acad Sci 1994;732:140-151.

56 Choi DH, Moon IS, Choi BK, Paik JW, Kim YS, Choi SH, Kim CK: Effects of sub-antimicrobial dose doxycycline on crevicular fluid MMP-8, and gingival tissue MMP-9, TIMP1 and IL- 6 levels in chronic periodontitis. J Periodont Res 2004:39:20-26.

57 Reddy MS, Geurs NC, Gunsolly JC: Periodontal host modulation with antiproteinase, anti-inflammatory, and bone-sparing agents: a systematic review. Ann Periodontol 2003;8:12-37.

58 Buduneli E, Vardar-Sengül S, Buduneli N, Atilla G, Wahlgren J, Sorsa T: Matrix metalloproteinases, tissue inhibitor of matrix metalloproteinase-1, and laminin-5 $\gamma 2$ chain immunolocalization in gingival tissue of endotoxin-induced periodontitis in rats: effects of low-dose doxycycline and aledronate. J Periodontol 2007;78:127-134.

59 Ciancio S, Ashley R: Safety and efficacy of sub-antimicrobial-dose doxycycline therapy in patients with adult periodontitis. Adv Dent Res 1998;12:27-31.

60 Golub LM, Lee HM, Stoner JA, Porsa T, Reinhardt RA, Wolff MS, Ryan ME, Nummikoski PV, Payne JB: Subantimicrobialdose doxycycline modulates gingival crevicular fluid biomarkers of periodontitis in postmenopausal osteopenic women. J Periodontol 2008;79:1409-1418.

61 De Paiva CS, Corrales RM, Villareal AL, Farley WJ, Li DQ, Stern ME, Pflugfelder SC: Corticosteroid and doxycycline suppress MMP-9 and inflammatory cytokine expression, MAPK activation in the corneal epithelium in experimental dry eye. Exp Eye Res 2006;83:526-535
62 Kim HS, Luo L, Pflugfelder SC, Li DQ: Doxycycline inhibits TGF- $\beta$-induced MMP-9 via SMAD and MAPK pathways in human corneal epithelial cells. Invest Ophthalmol Vis Sci 2005;46:840-848.

63 Määtä M, Kari O, Tervahartiala T, Peltonen S, Kari M, Saari M, Sorsa T: Tear fluid levels of MMP-8 are elevated in ocular rosacea treatment effect of oral doxycycline. Graefes Arch Clin Exp Ophthalmol 2006;244:957962.

64 Corbitt CA, Lin J, Lindsey ML: Mechanisms to inhibit matrix metalloproteinase activity: where are we in the development of clinically relevant inhibitors? Recent Pat Anticancer Drug Discov 2007;2:135-145.

65 Smith VA, Khan-Lim D, Anderson L, Cook SD, Dick AD: Does orally administered doxycycline reach the tear film? Br J Ophthalmol 2008;92:856-859.

66 Pruzanski W, Greenwald RA, Street IO, Laliberte F, Stefanski E, Vadas P: Inhibition of enzymatic activity of phospholipase A2 by minocycline and doxycycline. Biochem Pharmacol 1992;44:1165-1170.

67 Mäkelä A, Kuusi T, Schröder T: Inhibition of serum phospholipase-A2 in acute pancreatitis by pharmacological agents in vitro. Scand J Clin Lab Invest 1997;57:401-407.

68 Kari O, Aho VV, Peltonen S, Kari M, Määtä M, Collan Y, Saari KM: Group IIA phospholipase $\mathrm{A}_{2}$ concentration of tears in patients with ocular rosacea. Acta Ophthalmol Scand 2005;83:483-486

69 Del Rosso JQ: A status report on the medical management of rosacea: focus on topical therapies. Cutis 2002;70:271-275.

70 Cazalis J, Bodet C, Gagnon G, Grenier D: Doxycycline reduces lipopolysaccharide-induced inflammatory mediator secretion in macrophage and ex vivo human whole blood models. J Periodontol 2008;79:1762-1768.

71 Chung DR, Lee YS, Lee SS: Kinetics of inflammatory cytokines in patients with scrub typhus receiving doxycycline treatment. J Infect 2008;56:44-50.

72 Gürkan A, Cinarcik S, Hüseyinov A: Adjunctive subantimicrobial dose doxycycline: effect of clinical parameters and gingival crevicular fluid transforming growth factor$\beta$ levels in severe, generalized chronic periodontitis. J Clin Periodontol 2005;32:244253.

73 Esterly NB, Koransky JS, Furey NL, Trevisan M: Neutrophil chemotaxis in patients with acne receiving oral tetracycline therapy. Arch Dermatol 1984;120:1308-1313.

74 Gabler WL: Fluxes and accumulation of tetracyclines by human blood cells. Res Commun Chem Pathol Pharmacol 1991;72:3951

75 Gabler WL, Creamer HR: Suppression of human neutrophil functions by tetracyclines. J Periodont Res 1991;26:52-58.
76 Gabler WL, Tsukuda N: The influence of divalent cations and doxycycline on iodoacetamide-inhibitable leukocyte adherence. Res Commun Chem Pathol Pharmacol 1991;74: 131-140.

77 Webster GF, Toso SM, Hegemann L: Inhibition of a model of in vitro granuloma formation by tetracyclines and ciprofloxacin: involvement of protein kinase C. Arch Dermatol 1994;130:748-752.

78 Bakar O, Demircay Z, Yuksel M, Haklar G, Sanisoglu Y: The effect of azithromycin on reactive oxygen species in rosacea. Clin Exp Dermatol 2007;32:197-200.

79 Nielsen F, Mikkelsen BB, Nielsen JB, Andersen HR, Grandjean P: Plasma malondialdehyde as biomarker for oxidative stress: reference interval and effects of life-style factors. Clin Chem 1997;43:1209-1214.

80 Tisma VS, Basta-Juzbasic A, Jaganjac M, Brcic L, Dobric I, Lipozencic J, Tatzber F, Zarkovic N, Poljak-Blazi M: Oxidative stress and ferritin expression in the skin of patients with rosacea. J Am Acad Dermatol 2009;60: 270-276.

81 Miyachi Y, Yoshioka A, Imamura S, Niwa Y: Effect of antibiotics on the generation of reactive oxygen species. J Invest Dermatol 1986;86:449-453.

82 Akamatsu H, Asada M, Komura J, Asada Y, Nowa Y: Effect of doxycycline on the generation of reactive oxygen species: a possible mechanism of action of acne therapy with doxycycline. Acta Derm Venereol 1992;72: 178-179.

83 Amin AR, Attur MG, Thakker GD, Patel PD, Yvas PR, Patel RN, Patel IR, Abramson SB: A novel mechanism of action of tetracyclines: Proc Natl Acad Sci USA 1996;93:1301413019

84 Roméro-Graillet C, Aberdam E, Clément M, Ortonne JP, Ballotti R: Nitric oxide produced by ultraviolet-irradiated keratinocytes stimulates melanogenesis. J Clin Invest 1997;99: 635-642.

85 Amin AR, Patel RN, Thakker GD, Lowenstein CJ, Attur MG, Abramson SB: Posttranscriptional regulation of inducible nitric oxide synthase mRNA in murine macrophages by doxycycline and chemically modified tetracyclines. FEBS Lett 1997;410:259264.

86 Gomaa AH, Yaar M, Eyada MM, Bhawan J: Lymphangiogenesis and angiogenesis in non-phymatous rosacea. J Cutan Pathol 2007;34:748-753.

87 Lee CZ, Xu B, McCulloch CE, Yang GY, Young WL: Doxycyline suppresses cerebral matrix metalloproteinase- 9 and angiogensis induced by focal hyperstimulation of vascular endothelial growth factor in a mouse model. Stroke 2004;35:1715-1719.

88 Yao JS, Shen F, Young WL, Yang GY: Comparison of doxycyline and minocycline in the inhibition of VEGF-induced smooth muscle cell migration. Neurochem Int 2007; 590:524-530. 